\title{
Study on design of temperature - dependence of sublimation transfer roller
}

\author{
Ming-Chu Hsieh ${ }^{1, *}$ and Jung-Sen Lin $^{2}$ \\ ${ }^{1}$ Department of Mechanical Engineering, National Chin-Yi University of Technology, Taiwan \\ ${ }^{2}$ Department of Mechanical Engineering, National Chin-Yi University of Technology, Taiwan
}

\begin{abstract}
Sublimation trasnfer machine's hot roller, a compression type electronic heater, is made of ceramic core which is wrapped with nickelchromium alloy heater. This roller is insulated with magnesium oxide. When the power is on, the heat is generated. Heat transfers are from high temperature area to low temperature area, and it causes the non-uniform temperature surface and the roller surface distorsion. Such effects always increase with larger diameter of the roller and longer the roller. In order to predict the sublimation transfer machine's roller temperature distribution and validate it, we construct the roller's 3D temperature model and use ANSYS steady state heat transfer analysis module to calculate the power dissipation and simulate it. Final data shall help us to design sublimation transfer machines' roller.
\end{abstract}

\section{Introduction}

The phenomenon whereby that the solid materials are directly transferred into the gaseous phase by bypassing the liquid phase at a certain temperature and pressure are called sublimation. Sublimation transfer printing is a technology used to enable the direct transfer of dyes in sublimation inks from the solid into the gaseous phases by heating and pressurizing, then through the condensation in the solid phase to form graphics and text. Correspondingly, this technology is also known as gas transfer printing [1]. During the process of sublimation transfer printing, only the color of the dye will be transferred to produce graphics and text, while other resin substances in the sublimation ink stay attached to the film or paper of the carrier. The "functional intermediate carrier" only contains the printed graphics and text from the sublimate inks, and does not contain release and adhesive layers. Therefore, there are no detachable protective and bonding layers when the printed materials are transfer-printed [2]. This is the difference between sublimation transfer printing and other forms of thermal transfer printing. Since only the dye will be condensed on the surface of printed materials, the printed material always maintains its original characteristics after sublimation. For example, clothes can still hold their original soft nature after sublimation transfer printing. In addition to fabric, the transfer printing can also be applied to ceramics, metals, and other products. However, the printed materials have to possess heat resistance and the gap layer to accommodate dye molecules. During the sublimation transfer printing process, the printing layer and the receiving and accommodation layers of the printed materials must be kept under the compact attaching pressure to ensure good transferred printed graphics and text [3].

This study aims to the electrically heated hot roller's design and the calculation about power to keep well the hot roller's shell mechanism, then have a good allowable thermal

*Corresponding author: smj@ncut.edu.tw 
deformation of the roller. Therefore, for effectively controlling the heat transfer process, we need to analyze and calculate its heat resistance during heat conduction process. And then, we use finite element analysis software to determine the appropriate electric heaters' equipped position through uniform temperature analyses and simulations. During heat process, we first apply power. Heaters' temperature rise up gradually, and heat transfers from them to others where temperature are lower. Before reaching constant temperature, it's said to be transient heat conduction. When reaching constant temperature, i.e. reaching a complete thermal equilibrium, that is the heat in equals the heat out, it enters steady-state heat conduction [4].

Heater rollers' surface temperature and working time do a significant impact on the fabric printing quality [5]. While power on, the heaters' temperature reaches their maximum value immediately and rollers' other portion is still on low temperature, it causes thermal deformation longitudinally and radially and has a significant impact on the fabric printing quality. How to improve it? We'll provide practical data to support all of this report's objective.

\section{Basic theory}

\subsection{Heat sublimation transfer printing rollers}

\subsubsection{Heat transfer principle [6]}

All heat transfer modes require temperature differences, since heat transfers from high to low temperature area.

Conduction is effectively an energy transfer process from a matter particle with a higher energy to an adjacent matter particle with a lower energy, which rises the interaction between particles.

Fourier heat conduction equation:

$$
\begin{gathered}
q=\frac{Q_{\text {cond }}}{A}=-k \frac{d T}{d x} \\
q_{1}=\frac{Q_{\text {cond }}}{A}=-k \nabla T
\end{gathered}
$$

In equations (1) and (2), $q$ and $q_{1}$ are the heat values derived within a unit time period per unit area known as heat fluxes, $Q_{\text {cond }}$ is the thermal conductivity rate, $A$ is the surface area, $k$ is the coefficient of thermal conductivity for the material known as the thermal conductivity. where

\begin{tabular}{|l|l|}
\hline mark & description \\
\hline$q, q_{1}$ & $\begin{array}{l}\text { heat values derived per unit time, unit area and known as heat } \\
\text { fluxes. }\end{array}$ \\
\hline Qcond & thermal conductivity rate \\
\hline$A$ & surface area \\
\hline$k$ & $\begin{array}{l}\text { coefficient of thermal conductivity for the material known as } \\
\text { thermal conductivity }\end{array}$ \\
\hline$d T / d x$ and $\nabla \mathrm{T}$ & $\begin{array}{l}\text { temperature increments per unit length along the heat transfer } \\
\text { direction, known as the temperature gradient }\end{array}$ \\
\hline
\end{tabular}

From equation (1), thermal resistance can be represented as: 


$$
Q_{\text {cond }}=\frac{T_{1}-T_{2}}{R_{\text {cond }}}
$$

and

$$
R_{\text {cond }}=\frac{L}{k A}
$$

where $T_{1}-T_{2}$ is the temperature difference, $R_{\text {cond }}$ is the conduction resistance, and $L$ is the disatnce between two points where temperature difference occurs.

Convection refers to the energy transfer between the solid surface and the adjacent moving liquid or gas. It is only transferred between the solid surface and adjacent liquid via simple thermal conduction when the fluid flow is insufficient. Thus, the convection simultaneously combines fluid flow and heat conduction.

Newton's law of cooling:

$$
\begin{gathered}
q=\frac{Q_{\text {cond }}}{A}=h\left(h_{s}-T_{f}\right) \\
h=\frac{Q_{\text {conv }}}{A\left(T_{s}-T_{f}\right)}
\end{gathered}
$$

In equation (5), $\mathrm{q}$ is the heat flux, $Q_{\text {conv }}$ is the convective heat transfer rate, $T s-T_{f}$ is the temperature difference between the solid surface and fluid, and $h$ is the convective heat transfer coefficient defined as the heat transfer rate per unit area between the solid surface and the fluid per unit of temperature, which is affected by various factors, such as fluid properties, fluid velocity, wall shape, and roughness.

From equation (5), the concept of thermal resistance can be represented as,

$$
Q_{\text {conv }}=\frac{T_{s}-T_{f}}{R_{\text {conv }}}
$$

and

$$
R_{\text {cond }}=\frac{1}{h A}
$$

where $R_{\text {conv }}$ is the convection thermal resistance, 1 is the temperature difference between the two ends.

\subsubsection{Heat transfer equation}

Energy equation:Energy input - Energy output + Energy generated within the unit element $=$ Energy change per unit element.

$$
\left(q_{x 1}+q_{y 1}+q_{z 1}\right)-\left(q_{x 2}+q_{y 2}+q_{z 2}\right)+\rho^{\prime}\left(\Delta_{x} \Delta_{y} \Delta_{z}\right)=\rho\left(\Delta_{x} \Delta_{y} \Delta_{z}\right) C_{P} \frac{\Delta T}{\Delta t}
$$

where, $q_{x 1}, q_{y 1}, q_{z 1}$ are heat input transfer rate, $q_{x 2}, q_{y 2}$, and $q_{z 2}$, are output transfer rates, and $q^{\prime}$ is the energy produced per unit volume per unit time ; $\rho$ is the density

; $C_{p}$ is isobaric specific heat. 
Solid heat transfer equation:

$$
\begin{aligned}
& \rho C_{P} \frac{\partial T}{\partial t}=Q+\frac{\partial}{\partial x}\left(k \frac{\partial T}{\partial x}\right)+\frac{\partial}{\partial y}\left(k \frac{\partial T}{\partial y}\right)+\frac{\partial}{\partial z}\left(k \frac{\partial T}{\partial z}\right) \\
& \rho C_{P} \frac{\partial T}{\partial t}=Q+\nabla \cdot(k \nabla T)
\end{aligned}
$$

Equation (11) is the heat transfer equation derived from the energy conservation law and Fourier's law. The left side of the equation is the energy change. The second item in the right side of equation is the difference of heat transfer rates between input and output. Correspondingly, $Q$ is the heat source, $k$ is the thermal conductivity, $T$ is the temperature, and $t$ is time.

Fluid heat transfer equation:

$$
\begin{gathered}
\rho C_{P} \frac{\partial T}{\partial t}+\rho C_{P}\left(v_{x} \frac{\partial T}{\partial t}+v_{y} \frac{\partial T}{\partial y}+v_{z} \frac{\partial T}{\partial z}\right)=\frac{\partial}{\partial x}\left(k \frac{\partial T}{\partial x}\right)+\frac{\partial}{\partial y}\left(k \frac{\partial T}{\partial y}\right)+\frac{\partial}{\partial z}\left(k \frac{\partial T}{\partial z}\right)+Q \\
\rho C_{P} \frac{\partial T}{\partial t}+\nabla \cdot\left(-k \nabla T+\rho C_{P} T_{v}\right)=Q
\end{gathered}
$$

where $v_{x}, v_{y}$, and $v_{z}$, are the speed values along the directions of the $\mathrm{x}, \mathrm{y}$, and $\mathrm{z}$-axes, respectively, and $Q$ is the heat source. In equation (13), the symbols within the brackets denote the heat flow vector that includes the items of diffusion and convection.

Within the research context of this study, as the fluid was assumed to be in a steady flow, $\frac{\partial T}{\partial t}=0$ and the solid radioactive heat source $Q=0$. These were substituted into the solid heat transfer equation (11) that yields equation (14),

$$
\nabla \cdot(k \nabla T)=0
$$

Since $\frac{\partial T}{\partial t}=0$, and the fluid convection heat source $Q=0$, equation (15) can be obtained from the fluid heat transfer equation (13) as follows:

$$
\nabla \cdot\left(-k \nabla T+\rho C_{P} T_{v}\right)=0
$$

\subsection{Electric heater principle}

\subsubsection{The working principle of electric heater [7]}

Electric heater supplies heat by electric energy conversion. In the actual work, lowtemperature fluid flows into the input port through the pipeline at a certain pressure, and heated by electric energy supplied within the pipelines. All the high-temperature thermal energy produced from the electric heat source through this path is required in accordance to the thermodynamics principles for fluids. Therefore, the temperature of the heated medium should increase. According to the signals of the temperature sensor at the output port, the internal control system of the electric heating tubes automatically adjusts the heat output power of the electric heating tubes to keep uniform medium temperature at the output port. 
When the heating element is overheated, the independent overheating protection device of the heating element would immediately cut off the heating power to prevent the coking, deterioration, and carbonization of heating materials, as well as the serious burnout of heating elements caused by overheating. This would effectively prolong the service life of the electric heater.

\subsubsection{Electric heater design and calculation}

Generally, the design and calculation of electric heating are divided into the following three parts:

1. Calculate the power consumption for heating from the initial state to attain a specific temperature within the required time a specific temperature at required time

2. Calculate the thermal energy and power required to keep the medium temperature constant.

3. The total power assigned to is the sum of above two maximum vales times 1.2 for safe operation.

(1) The power consumption required to maintain the heating at the initial stages is,

$$
K_{w}=(C 1 M 1 \Delta T+C 2 M 2 \Delta T) / 863 / H+P / 2
$$

where $P$ is the heat dissipation of the container at the final temperature (unit, $\mathrm{kW}$ ), $H$ is the time required for heating from the initial temperature to the final required temperature (unit, h), $\mathrm{M}_{1}$ and $\mathrm{M}_{2}$ are the masses of the container and medium, respectively, (units, $\mathrm{kg}$ ), $\mathrm{C}_{1}$ and $\mathrm{C}_{2}$, the specific heat capacities of the container and medium, respectively, (units, $\mathrm{kcal} /\left(\mathrm{kg} \cdot{ }^{\circ} \mathrm{C}\right)$, and $\Delta \mathrm{T}$ is the temperature difference between the final and initial temperatures (unit ${ }^{\circ} \mathrm{C}$ ).

(2) The required power consumption for maintaining the medium temperature is defined as,

$$
K_{w}=\mathrm{C} 2 \mathrm{M} 3 \Delta \mathrm{T} / 863+P
$$

where $\mathrm{M}_{3}$ is the mass flow per hour (unit, $\mathrm{kcal} / \mathrm{h}$ ).

\section{Methods}

\subsection{Experimental steps}

\subsubsection{The mechanism of heat sublimation transfer printing rollers}

All heat printing rollers should include the workbench control box, transport equipment, electrically heated hot roller mechanism, detection devices, support tripod, and other parts, as shown in Figure 1. 
1. Workbench :The workbench is used to hold fixtures and printing stocks

2. Control system: The control system uses a microcomputer to control the length of the processing roll, and achieve the purpose of accurate material feeding to the machine

3. Conveyor system: Feeding and receiving equipments are also used

4. Electrically heated hot roller: Hot pressing device is a heated roller appliance that uses arc surface rolling to produce hot forming

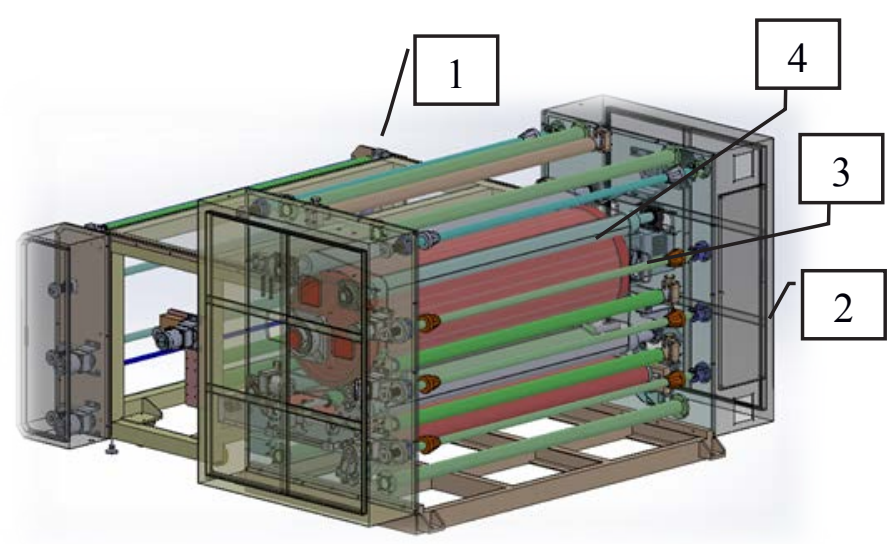

Fig. 1. Heat Sublimation Transfer Printing Rollers basic structure.

\subsubsection{Hot rollers' design for sublimation heat printing}

For the design of the hot roller for sublimation heat printing, drawing software was used to illustrate the external dimensions of the hot roller structure and the arrangement mode of the electric heaters, is shown in Fig. 2 shows the hot rollers' structure and the arrangement of heaters.
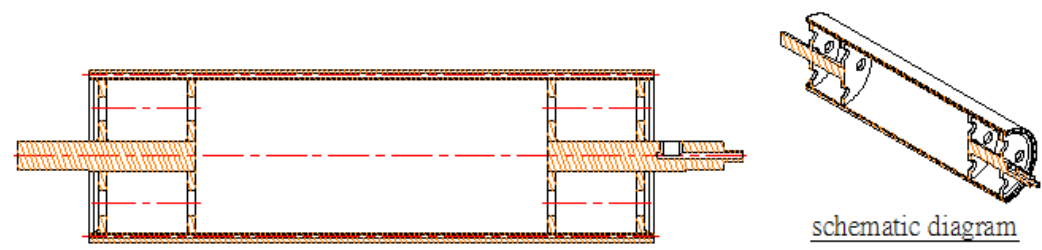

$(2: 1)$
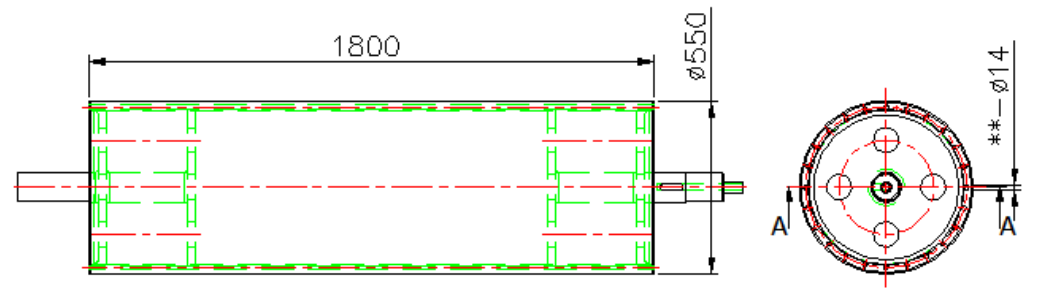

Fig. 2. Design of the hot roller for sublimation heat printing. 


\subsubsection{Model establishment}

The drawing software, SolidWorks, was used to draw the geometric schematic of the building structure. It was then imported into the software ANSYS for the numerical analysis of the heat flux field of the hot roller. The solution process designed by the study is shown in Figure 3.

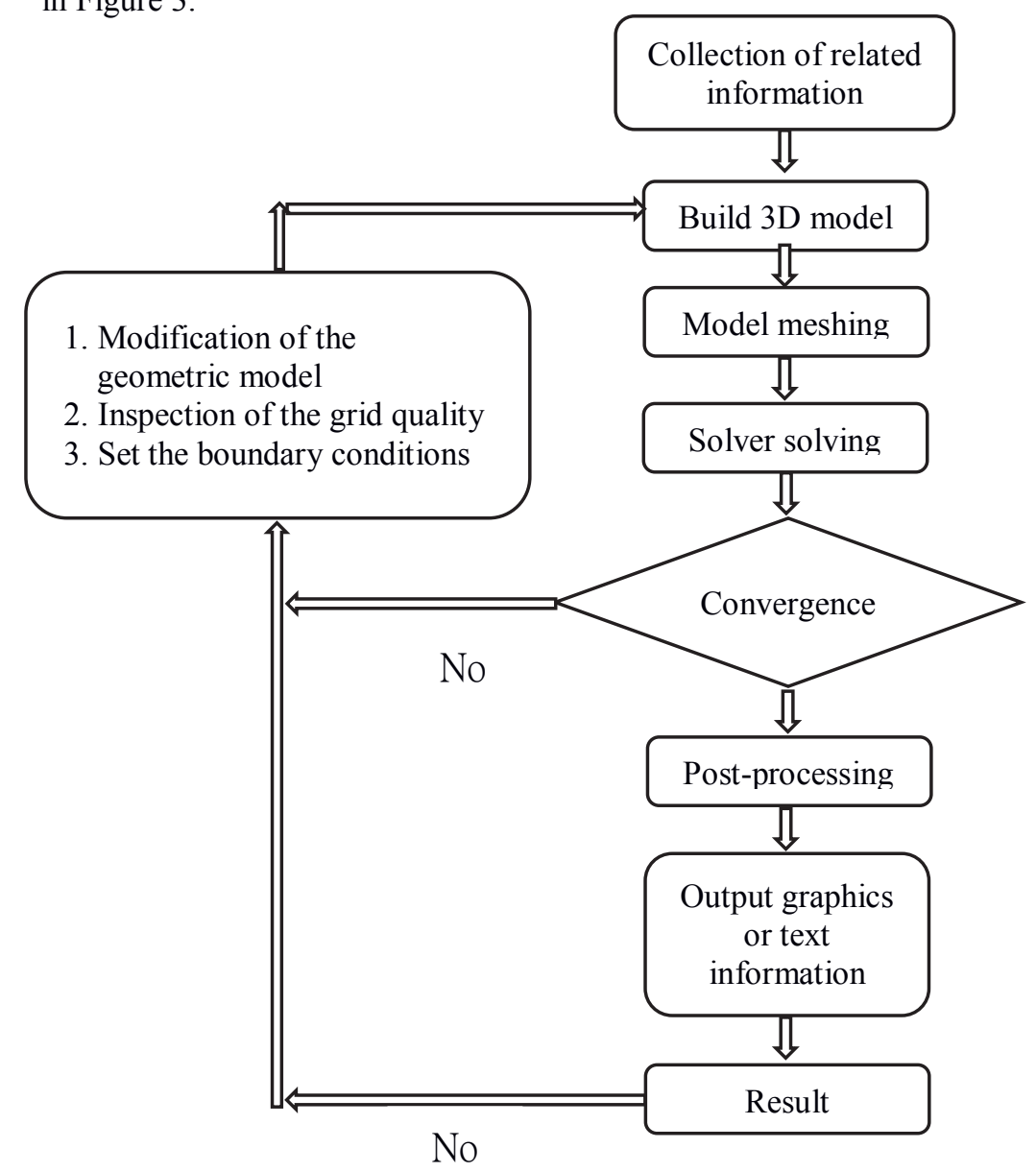

Fig. 3. Flow chart of simulation analysis and design.

\subsubsection{Assumptions, equations and boundary conditions}

As shown in Figure 4, the electric heater and the heated roller are joined, and heat will be transferred by heat conduction. In this study, we assume it's a steady-state, laminar flow field and the fluid is incompressible. . The buoyancy effect of the air layer caused by the temperature difference in the presence of a gravitational field was also considered. The ambient temperature was set to $25^{\circ} \mathrm{C}$. Its numerical simulation included a natural convection and heat conduction, while the effect of thermal radiation was ignored [8]. 

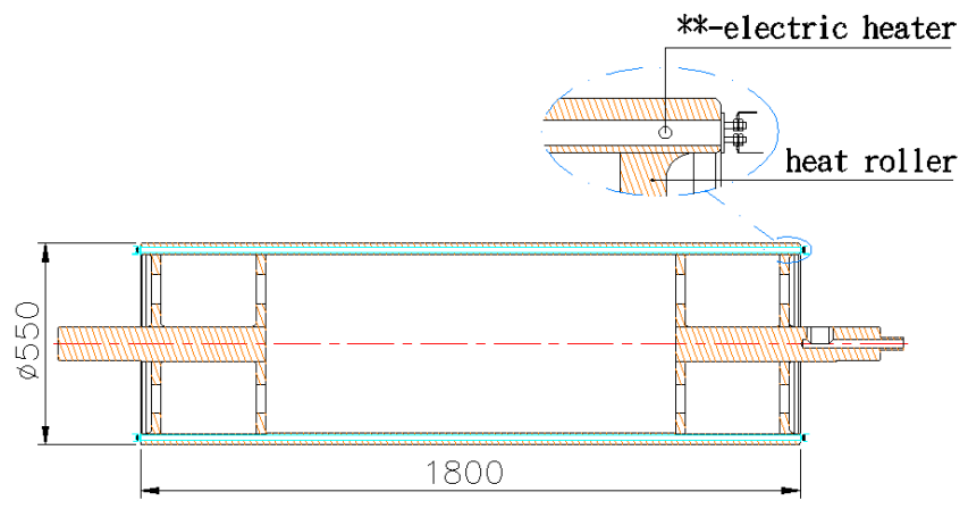

Fig. 4. Module diagram of hot roller and electric heater.

The specific details pertaining to the boundary conditions are as follows:

(1) The thickness of the air layer: The gap thickness between the electric heater and the hot roller was only $0.15 \mathrm{~mm}$, and was integrated with the atmospheric layer. With the specific thickness, if the number of the resolution grid we used is too large, there may be no sufficient computer memory to perform this simulation. If the model grid is not fine enough, the calculation results would be affected. In addition, this gap acts as of thermal resistance. Moreover, the gap would be smaller in the case of thermal expansion. So, we can have a good reasonable simplification.

(2) The thermal conductivity coefficient of air on the outer surface of the hot roller was $15 \mathrm{~W} / \mathrm{m}^{2} \cdot{ }^{\circ} \mathrm{C}$

(3) The initial temperature on the hot roller was set to $25^{\circ} \mathrm{C}$

(4) The heat flux of the electrical heaters on the hot roller were calculated by two different calorific value calculations and analyses, in accordance to the products of $24 \times 800 \mathrm{~W}$ and $12 \times 1600 \mathrm{~W}$

(5) Properties of steel

Name: Hot Roller

Material: SS400

Density: $7850 \mathrm{~kg} / \mathrm{m}^{3}$

Specific heat: $480 \mathrm{~J} / \mathrm{kg} \cdot \mathrm{K}$

Thermal conductivity: $48 \mathrm{~W} / \mathrm{m} \cdot \mathrm{K}$

(6) Solution: In accordance to the steady-state heat conduction, the system would achieve equilibrium temperature after some time.

\section{Results and discussions}

\subsection{Simulation results and discussions}

ANSYS provides a cyclic process that includes a series of analyses, evaluations, and modifications. Correspondingly, we analyzed the initial design, and evaluated the analyzed results according to the design requirements, followed by a subsequent modification of the design. This cyclic process would execute repeatedly until all the requirements were met.

\subsubsection{Effect of piping arrangement of electric heater}

Fig. 5 shows the temperature distribution of the hot roller, temperatures on both ends are the lowest because of the air convection occurs mainly on both ends. Thus, the temperature 
of the middle part was higher than the temperatures of the left and right sides of the inlet and outlet. This was owing to the larger thermal resistance required for conduction in the middle area of the hot roller, and the relatively long distance to the edge of the hot roller. This distance elicited a relatively slow heat transfer from the middle to the two sides, and thus yielded a higher temperature in the middle part in Figure 5.

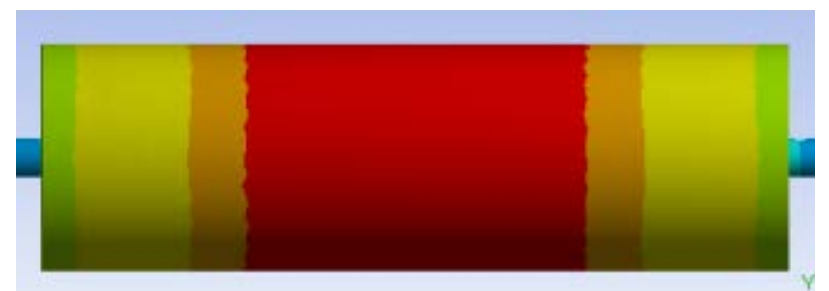

Fig. 5. Temperature distribution of the hot roller.

\subsubsection{Effect of pipe spacing}

The processing pipeline spacing of the hot roller electric heater is shown Figure 6. The pipe spacing between the inlet and outlet was $132.5 \mathrm{~mm}$. As shown in the temperature and thermal conduction distribution of the hot roller, the solid heat transfer rate was reduced. This effect was attributed to the oversized pipe spacing, thus giving rise to a modest uniformity in terms of the temperature distribution. When the pipe spacing for the inlet and outlet pipes was adjusted to $66.5 \mathrm{~mm}$, its heat conduction and temperature distributions are modified in accordance to those shown in Figure 7, whereby the uniformity of the temperature distribution for the hot roller was significantly improved given the same heat flux.

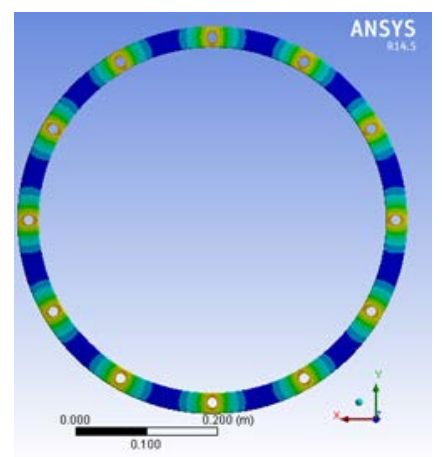

Fig. 6. Temperature profile of left and right sides of the electrically heated hot roller (12 holes). 


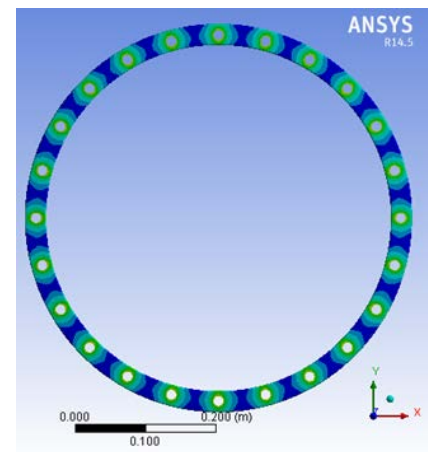

Fig. 7. Temperature profile of left and right sides of the electrically heated hot roller (24 holes).

\subsubsection{Performance effect of temperature uniformity}

From the beginning of heating to the final working temperature, the electrically heated hot roller has yielded optimum temperature uniformity along the axial direction. At increased temperatures, the effects of the uniform temperatures became more pronounced. Therefore is shown Figure 8-9, the locations and numbers of the electric heaters have an important effect on the temperature distribution of the heat rollers.

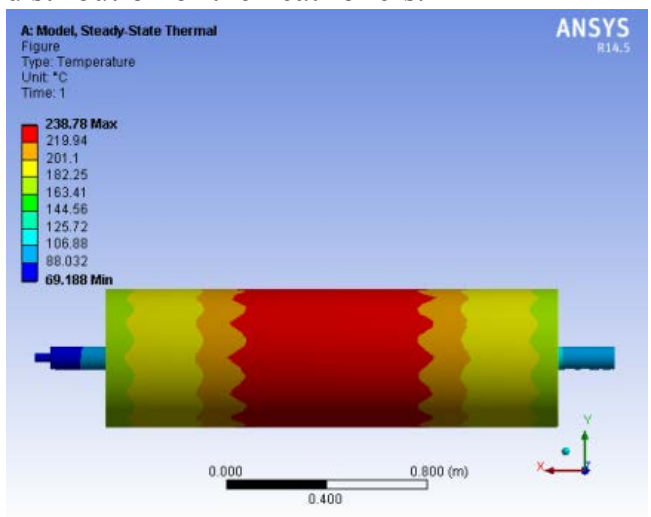

Fig. 8. Temperature profile of the electrically heated hot roller ( 12 holes with 12 heaters).

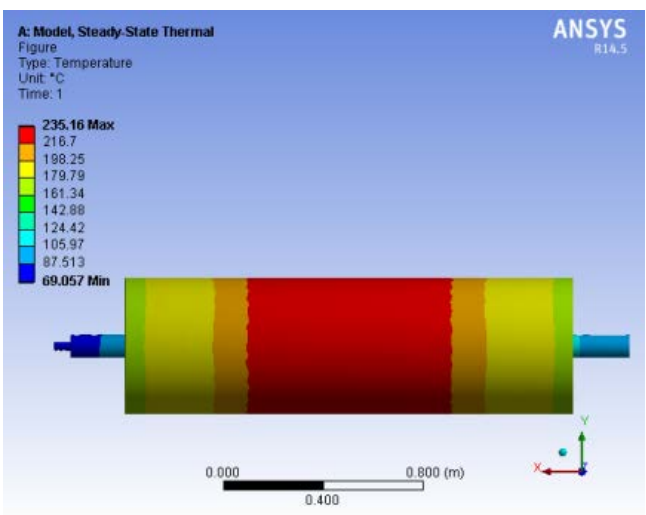

Fig. 9. Temperature profile of the electrically heated hot roller ( 24 holes with 24 heaters). 


\subsection{Temperature simulation results for different pipe spacing arrangements on the hot roller}

\subsubsection{Roller with 12 heaters}

This section illustrates the simulation of temperature data at different positions of the electrically heated hot rollers. The positions of the electrically heated hot rollers (12 holes with 12 heaters) are shown in Figure 10. The dimensions of the hot roller model were 1800 $\mathrm{mm}(\mathrm{L}) \times 550 \mathrm{~mm}(\mathrm{D})$. The locations are sequentially numbered from 1 to 28 from the right part of the schematic to the left. The simulation results of the temperature distribution are shown in Table 1.

The temperature difference at section $\mathrm{A}$ of the hot roller $=235.99-230.67=5.32^{\circ} \mathrm{C}$ The temperature difference at section $\mathrm{B}$ of the hot roller $=220.05-219.63=0.43^{\circ} \mathrm{C}$ The temperature difference at section $\mathrm{C}$ of the hot roller $=202.26-200.53=1.73^{\circ} \mathrm{C}$ The temperature difference at section $\mathrm{D}$ of the hot roller $=182.24-181.01=1.23^{\circ} \mathrm{C}$
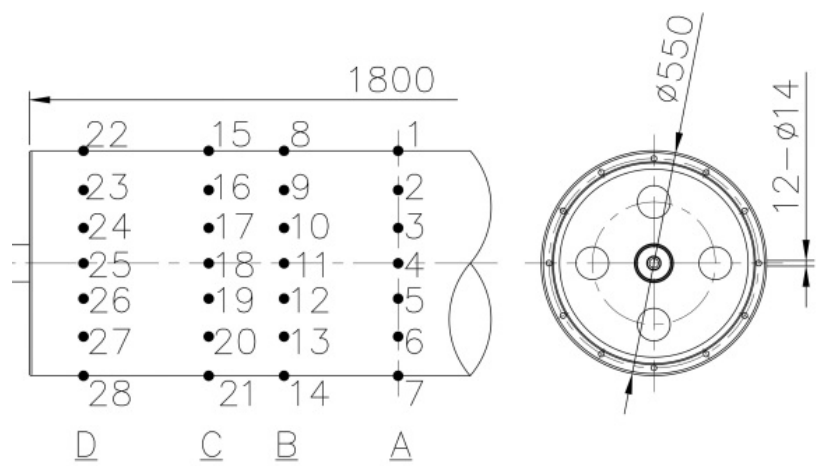

Fig. 10. Position distribution for temperature profile of electrically heated hot roller (12 holes).

Table 1. Temperature profile of the front model (12 holes).

\begin{tabular}{|c|c|c|c|c|c|c|c|}
\hline Section A & $\mathbf{1}$ & $\mathbf{2}$ & $\mathbf{3}$ & $\mathbf{4}$ & $\mathbf{5}$ & $\mathbf{6}$ & $\mathbf{7}$ \\
\hline Temperature & 231.02 & 231.43 & 230.98 & 235.99 & 230.67 & 233.55 & 231.5 \\
\hline Section B & $\mathbf{8}$ & $\mathbf{9}$ & $\mathbf{1 0}$ & $\mathbf{1 1}$ & $\mathbf{1 2}$ & $\mathbf{1 3}$ & $\mathbf{1 4}$ \\
\hline Temperature & 219.84 & 220.05 & 219.77 & 219.85 & 219.66 & 219.63 & 219.85 \\
\hline Section C & $\mathbf{1 5}$ & $\mathbf{1 6}$ & $\mathbf{1 7}$ & $\mathbf{1 8}$ & $\mathbf{1 9}$ & $\mathbf{2 0}$ & $\mathbf{2 1}$ \\
\hline Temperature & 202.26 & 200.53 & 200.99 & 200.5 & 201.25 & 200.86 & 201.01 \\
\hline Section D & $\mathbf{2 2}$ & $\mathbf{2 3}$ & $\mathbf{2 4}$ & $\mathbf{2 5}$ & $\mathbf{2 6}$ & $\mathbf{2 7}$ & $\mathbf{2 8}$ \\
\hline Temperature & 181.94 & 182.21 & 182.24 & 181.01 & 181.93 & 181.79 & 181.3 \\
\hline
\end{tabular}

\subsubsection{Roller with 24 heaters}

This section illustrates the simulation data of the temperature on different positions of the electrically heated hot roller. The positions used for the evaluation the temperature distribution of the electrically heated hot roller (24 holes) are shown in Figure 11. The dimensions of the hot roller model were $1800 \mathrm{~mm}(\mathrm{~L}) \times 550 \mathrm{~mm}$ (D). The locations are sequentially numbered from 1 to 28 from the right part to the left. The simulation results of the temperature distribution are listed in Table 2.

The temperature difference at section $\mathrm{A} 1$ of the hot roller $=233.81-233.07=0.74^{\circ} \mathrm{C}$ 
The temperature difference at section $\mathrm{B} 1$ of the hot roller $=217.03-216.20=0.83^{\circ} \mathrm{C}$

The temperature difference at section $\mathrm{C} 1$ of the hot roller $=198.45-197.29=1.16{ }^{\circ} \mathrm{C}$

The temperature difference at section D1 of the hot roller $=179.87-179.30=0.57 \quad{ }^{\circ} \mathrm{C}$
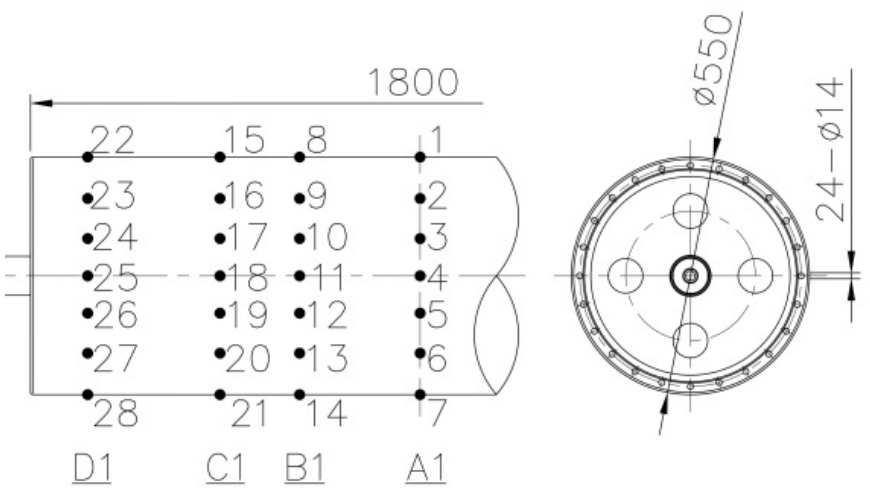

Fig. 11. Position distribution for temperature profile of electrically heated hot roller ( 24 holes).

Table 2. Temperature profile of the front model (24 holes).

\begin{tabular}{|c|c|c|c|c|c|c|c|}
\hline Section A1 & $\mathbf{1}$ & $\mathbf{2}$ & $\mathbf{3}$ & $\mathbf{4}$ & $\mathbf{5}$ & $\mathbf{6}$ & $\mathbf{7}$ \\
\hline Temperature & 233.81 & 233.07 & 233.73 & 233.6 & 233.48 & 233.45 & 233.69 \\
\hline Section B1 & $\mathbf{8}$ & $\mathbf{9}$ & $\mathbf{1 0}$ & $\mathbf{1 1}$ & $\mathbf{1 2}$ & $\mathbf{1 3}$ & $\mathbf{1 4}$ \\
\hline Temperature & 216.86 & 216.26 & 216.2 & 216.65 & 216.7 & 216.2 & 217.03 \\
\hline Section C1 & $\mathbf{1 5}$ & $\mathbf{1 6}$ & $\mathbf{1 7}$ & $\mathbf{1 8}$ & $\mathbf{1 9}$ & $\mathbf{2 0}$ & $\mathbf{2 1}$ \\
\hline Temperature & 198.22 & 197.36 & 197.61 & 198.39 & 197.29 & 198.08 & 198.45 \\
\hline Section D1 & $\mathbf{2 2}$ & $\mathbf{2 3}$ & $\mathbf{2 4}$ & $\mathbf{2 5}$ & $\mathbf{2 6}$ & $\mathbf{2 7}$ & $\mathbf{2 8}$ \\
\hline Temperature & 179.77 & 179.3 & 179.69 & 178.81 & 178.58 & 178.37 & 179.87 \\
\hline
\end{tabular}

\subsubsection{Simulation data for temperature difference with 12 and 24 heaters}

For the same heat flux, the effects of pipe spacing which changes the temperature uniformity of the hot roller are shown in Table 3. Elicited results show that the temperature difference became smaller as the spacing changed.

Table 3. Temperature difference for different pipe spacings of hot roller.

\begin{tabular}{|l|c|c|}
\hline $\begin{array}{l}\text { Position for the temperature on hot } \\
\text { roller }\left({ }^{\circ} \mathrm{C}\right)\end{array}$ & $\begin{array}{l}\text { Temperature profile of the } \\
\text { electrically heated }(12 \text { holes })\end{array}$ & $\begin{array}{l}\text { Temperature profile of the } \\
\text { electrically heated }(24 \text { holes })\end{array}$ \\
\hline $\begin{array}{l}\text { Maximum temperature difference } \\
\text { between A and A1 }\left({ }^{\circ} \mathrm{C}\right)\end{array}$ & 5.32 & 0.74 \\
\hline $\begin{array}{l}\text { Maximum temperature difference } \\
\text { between B and B1 }\left({ }^{\circ} \mathrm{C}\right)\end{array}$ & 0.43 & 0.83 \\
\hline $\begin{array}{l}\text { Maximum temperature difference } \\
\text { between C and } \mathrm{C} 1\left({ }^{\circ} \mathrm{C}\right)\end{array}$ & 1.73 & 1.16 \\
\hline $\begin{array}{l}\text { Maximum temperature difference } \\
\text { between D and D1 }\left({ }^{\circ} \mathrm{C}\right)\end{array}$ & 1.23 & 0.57 \\
\hline Total temperature difference $\left({ }^{\circ} \mathrm{C}\right)$ & 4.89 & 0.59 \\
\hline
\end{tabular}




\section{Conclusions}

Sublimation transfer printing technology is currently one of the most important fabric printing technologies in the fabric market. In manufacturing, it is impossible to obtain the best color quality at an inappropriate temperature. Correspondingly, printing with poor quality indirectly results in the bad printing and wasting of the materials in the absence of optimized data. The optimal benefits of the sublimation transfer printing can only be achieved with the combination of the optimized temperature uniformity and heating time. Therefore, in this study, the finite element method was used to study the temperature uniformities of under the various designs of electric heating pipes and hot rollers for with different number of heaters on roller at the same power consumption. As the pipe spacing on the hot roller increased, the heat transfer became poorer, and the temperature uniformity worsened. Smaller hot roller pipe spacing resulted in a better transfer efficiency, pipeline configuration, and power consumption, and therefore led to an increased temperature uniformity of the electrically heated hot roller. Using auxiliary design tool software, computer aided engineering analysis became practical. Engineers can use CAD and CAE software for predesign and development work, which could significantly reduce the time and cost of the development process, and provide an important design reference basis for design and development.

\section{References}

1. Y.M. Tsai, The Image Quality Assessment of Digital Transfer Printing, National Taiwan University of Arts/Graduate School of Applied Media Arts, New Taipei City (2006)

2. A.T. Hsiao, A Study of Color Quality Characteristics on Textile Using Different Dye Sublimation Processes, Chinese Culture University, Department of Information Communication, Taipei City (2014)

3. T.R. Chou and K. L. Hung, General Description of Thermal Printing Process and Development, 26, 1-115 (2010)

4. C.Y. Hsieh, K. M. Tsai, S. R. Juang, L. G. Chen, ANSYS Advanced Examples of Computer Aided Engineer Analysis, QUARK studio, Publisher Easy Book

5. J.H. Chien, New Ink Printing Technology: Beijing: China Light Industry Press, 2000 (in Chinese)

6. Y.A. Cengel, N. Yao, Heat Transfer: A Practical Approach, Liu He Publishing House (2000)

7. M.C. Cheng, The Study of Electric Heater Used in the Thermal Oil Boiler Operation, National Changhua Normal University, Department of Electrical Engineering, Changhua City (2012)

8. J.W. Lee, H.M. Hwang, and I.J. Chung, Finite-element Modeling and Analysis of Time-dependent Thermomechanical Distortion of Optical Sheets in an LCD module, Proceedings of the 6th International Meeting on Information Display and the $5^{\text {th }}$ International Display Manufacturing Conference, pp. 1436-1441 (2006) 